\title{
CCD272-64 and GSENSE400BSI- GP CMOS quantum efficiency measurement in EUV and VUV
}

\section{Andrey Shugarov, Eugene Vishnyakov, Anton Nikolenko, Denis Ivlyushkin, Pavel Zavertkin, et al.}

Andrey S. Shugarov, Eugene A. Vishnyakov, Anton D. Nikolenko, Denis V. Ivlyushkin, Pavel S. Zavertkin, Alexey S. Kirichenko, Sergey V. Kuzin, Andrey A. Pertsov, "CCD272-64 and GSENSE400BSI-GP CMOS quantum efficiency measurement in EUV and VUV," Proc. SPIE 11852, International Conference on Space Optics - ICSO 2020, 118525C (11 June 2021); doi: 10.1117/12.2599947

SPIE Event: International Conference on Space Optics - ICSO 2021, 2021, Online Only 


\section{International Conference on Space Optics-ICSO 2020}

Virtual Conference

30 March-2 April 2021

Edited by Bruno Cugny, Zoran Sodnik, and Nikos Karafolas
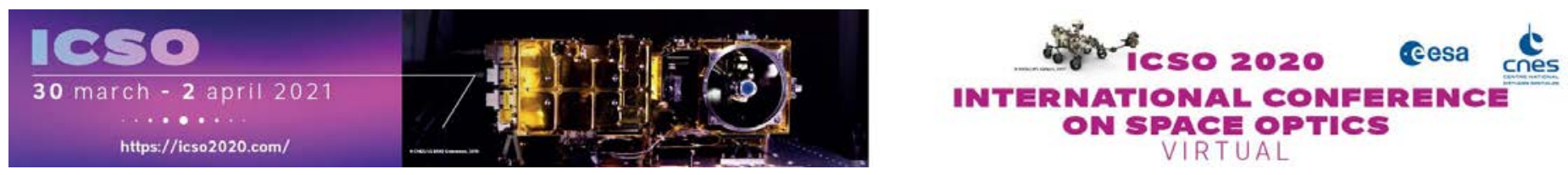

\section{CCD272-64 and GSENSE400BSI-GP CMOS quantum efficiency measurement in EUV and VUV}

\section{Cesa isoporeseatings denes}




\title{
CCD272-64 and GSENSE400BSI-GP CMOS quantum efficiency measurement in EUV and VUV
}

\author{
Andrey S. Shugarov*a, Eugene A. Vishnyakov ${ }^{\mathrm{b}}$, Anton D. Nikolenko ${ }^{\mathrm{c}}$, Denis V. Ivlyushkin ${ }^{\mathrm{c}}$, \\ Pavel S. Zavertkin ${ }^{c}$, Alexey S. Kirichenko ${ }^{c}$, Sergey V. Kuzin ${ }^{c}$, Andrey A. Pertsov ${ }^{c}$ \\ ${ }^{a}$ Institute of Astronomy of the Russian Academy of Sciences, 48 Pyatnitskaya Street, Moscow \\ 119017, Russian Federation; ${ }^{b}$ P.N.Lebedev Physical Institute of the Russian Academy of Sciences, \\ 53 Leninskiy Prospekt, Moscow 119991, Russian Federation; ${ }^{\mathrm{c}}$ Budker Institute of Nuclear Physics, \\ Russian Academy of Sciences, 11 Ac. Lavrentiev Prospekt, Novosibirsk 630090, Russian Federation
}

\begin{abstract}
We have measured the spectral quantum efficiency of several digital detectors in two spectral ranges, namely vacuum ultraviolet (VUV, 115-310 nm) and extreme ultraviolet (EUV, 10-58 nm) wavebands. We used monochromatic synchrotron radiation from the VEPP-4M storage ring (INP, Novosibirsk) to investigate the spectral response of GSENSE400BSI-GP CMOS which have been specially designed for optimum VUV sensitivity, as well as the WSO-UV project's custom deep cooled CCD272-64 sealed within a hermetic contaminant-protective stainless-steel enclosure with a VUV-transparent entrance window. The CMOS GSENSE400BSI-GP sensitive surface has four types of different pretreatment: (i) small boron implantation dose, weak annealing; (ii) small boron implantation dose, strong annealing, (iii) large boron implantation dose, weak annealing; (iv) large boron implantation dose, strong annealing. These VUV optimised CMOS sensors have higher spectral sensitivity and higher quantum efficiency in the 112-180 nm spectral range, compared to a commercial CMOS which is typically optimised for the best performance at visible wavelengths, but at longer wavelengths GSENSE400BSI-GP sensitivity decreases. The measurements have revealed that stronger annealing can slightly increase the CMOS spectral sensitivity, while large boron implantation dose decreases the sensitivity of the CMOS sensors. With relation to quantum efficiency figures, CCD still prevails in the VUV while recent advances in CMOS technology could make CMOS more preferable in the EUV range.
\end{abstract}

Keywords: quantum efficiency; vacuum ultraviolet; extreme ultraviolet; silicon detectors; CCD matrices; CMOS; synchrotron radiation; WSO-UV.

\section{INTRODUCTION}

Extraterrestrial UV astronomy has always required digital detectors for imaging and spectral data acquisition, enabling large total observation time to distinguish a vast range of phenomena occurring in stars, galaxies, nebulas and interstellar medium at different timescales, ranging from several seconds to several years. Contemporary scientific demands to be imposed on the new generation of digital detectors for space applications are challenging enough because they imply simultaneously low noise, wide dynamic range, high sensitivity of the detector to electromagnetic radiation of specific wavelengths, large sensitive operating area and high level of uniformity of these properties across the detector aperture.

The first integrating $\mathrm{p}-\mathrm{n}$ junction silicon photodetector ${ }^{1,2}$ was demonstrated by G. P. Weckler at the Fairchild Semiconductor company in 1965 . He noticed that a reverse biased silicon p-n junction in an integrated circuit could give a photocurrent under exposure to light, causing discharge of the voltage applied to the photodiode with accordance to its capacitance. Later this principle was successfully used in two different technologies of photodetectors production, namely CCD (charge coupled device) and CMOS (complementary metal-oxide-semiconductor) photodetector techniques. Great improvement in the reduction of the dark current due to the thermal generation of the charge carriers and their subsequent diffusion has been achieved in 1968 by P. J. W. Noble at Plessey where he had proposed a buried photodiode structure ${ }^{3}$.

The first CCD array ${ }^{4}$ was built in 1969 by W. S. Boyle and G. E. Smith at Bell Labs, and its application to image sensors has been rapidly recognized ${ }^{5,6}$. Later in 1973 , S. R. Shortes and his colleagues had developed a thinned backsideilluminated CCD imaging technique ${ }^{7,8}$. Since then, CCD production technology has evolved progressively ${ }^{6}$. 
On the contrary, the first CMOS active pixel image sensor with an intra-pixel charge transfer was introduced only in 1993 by E. R. Fossum ${ }^{9}$, with subsequent performance improvement using backside illumination ${ }^{10,11}$. Today, in 2021 , both CMOS and CCD technologies are developed enough to meet the requirements of photodetectors for astronomy.

In the current work, we compare the quantum efficiency of several CMOS and CCD image sensors in VUV and EUV spectral ranges. GSENSE400BSI-GP CMOS sensor is a special VUV-dedicated CMOS sensor produced by GPixel in China, while CCD272-64 is a semi-custom device produced by Teledyne e2v for specific astronomical UV observations.

\section{TECHNOLOGICAL PECULIARITIES OF THE DETECTORS}

The first type of the semiconductor devices under study is a custom sealed enclosure ${ }^{12,13}$ containing a CCD272-64 sensor which will be used as a UV photodetector in the WSO-UV (World Space Observatory - Ultraviolet) international space project $^{14,15}$. The dimensions of this CCD are $37.3 \times 49.1 \mathrm{~mm}$ (i. e. $3112 \times 4096$ square pixels of $12 \times 12 \mu \mathrm{m}$ size). The CCD has been optimized to simultaneously ensure a low readout noise $\sim 3 \mathrm{e}^{-}$and a low dark current $\sim 10 \mathrm{e}^{-} / \mathrm{pixel}^{\mathrm{h}}$ hour at an operating temperature of $-100{ }^{\circ} \mathrm{C}$. Part of the CCD sensitive surface is covered with antireflection coating to enhance the CCD sensitivity in the 170-310 nm spectral range. The effect of the antireflection coating on quantum efficiency has been experimentally measured ${ }^{16}$ and will be presented in detail elsewhere. The detector enclosure has a $\mathrm{MgF}_{2} \mathrm{spectral}$ filter window to absorb all the energetic VUV radiation with wavelengths shorter than $112 \mathrm{~nm}$. The outer look of the detector enclosure is presented in Fig. 1 . We have measured UV sensitivity of the whole detector with the $\mathrm{MgF}_{2} \mathrm{window}_{\text {. }}$
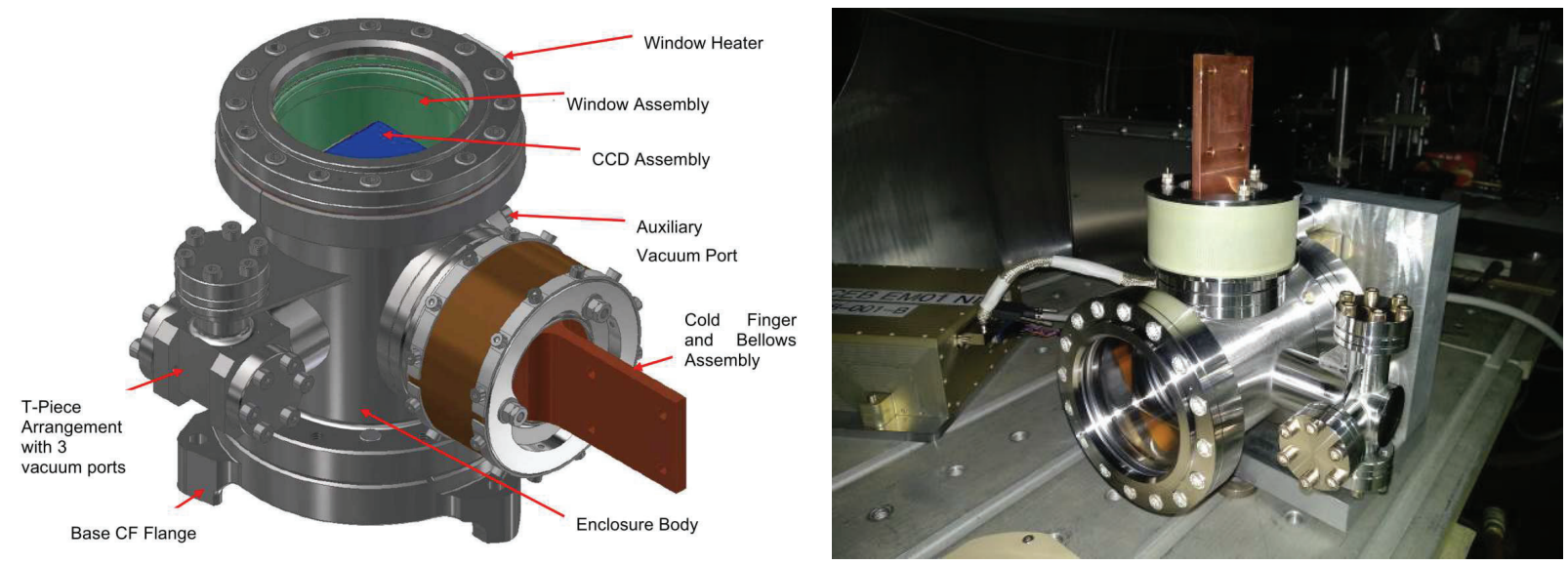

Figure 1. External view of the CCD-containing stainless-steel enclosure with $\mathrm{MgF}_{2}$ protective window and $\mathrm{Cu}$ Cold Finger.

The second type of semiconductor detectors under study is GSENSE400BSI-GP CMOS sensor that is a special VUVdedicated CMOS sensor operable at the room temperature, while CCD272-64 is a deep cooled digital detector with a working temperature of $-100^{\circ} \mathrm{C}$ on the sensitive surface of the CCD. All the CMOS sensors have a sensitive area of $22.528 \times 22.528 \mathrm{~mm}$, which contains $2048 \times 2048$ square pixels of $11 \times 11 \mu \mathrm{m}$ size.

The Chinese GPixel company has kindly provided us with two GSENSE400BSI-GP CMOS sensors divided into 2 parts each, with each part having a different treatment (see Table 1). The two CMOS sensors have different levels of boron implantation (Large or Small Boron implantation dose, LB and SB respectively), each CMOS sensor being thermally annealed at 2 different degrees: upper part with Weaker Annealing (WA), and lower part with Stronger Annealing (SA).

Table 1. Description of the GSENSE400BSI-GP CMOS sensor labels used in the current work.

\begin{tabular}{|c|c|c|}
\hline CMOS Part Label & Boron Ion Doping & Annealing Degree \\
\hline LB-SA & Large Boron Implantation Dose & Strong Annealing \\
\hline LB-WA & Large Boron Implantation Dose & Weak Annealing \\
\hline SB-SA & Small Boron Implantation Dose & Strong Annealing \\
\hline SB-WA & Small Boron Implantation Dose & Weak Annealing \\
\hline
\end{tabular}




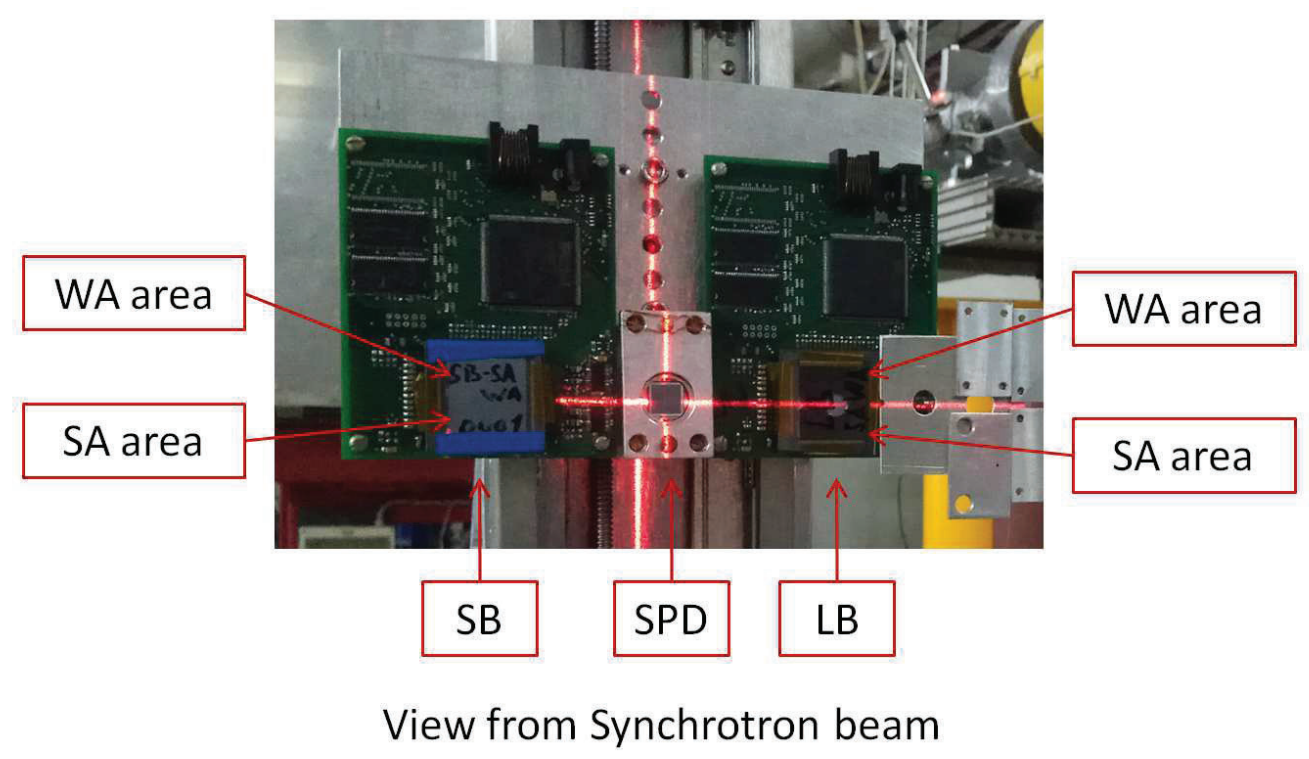

Figure 2. External view of the CMOS sensors in question mounted on a translation stage with a calibrated photodiode SPD.

The external view of the two GSENSE400BSI-GP CMOS sensors with drive electronics is presented in Fig. 2 . The designations WA/SA, SB/LB correspond to the label descriptions given in Table 1. Each CMOS sensor in Fig. 2 has a protective glass cover with designations. Between the two CMOS sensors in Fig. 2 there is a calibrated detector SPD, which was used in the experiment series as a reference detector. A detailed description is given in the next section.

\section{EXPERIMENTAL MOUNTING AND PROCEDURES}

The experimental measurements were taken at Kosmos Metrological Station ${ }^{17}$ at Budker Institute of Nuclear Physics in Novosibirsk, Russia. The station is useful for absolute calibration of detectors like CMOS or CCD sensors in the ultraviolet (UV), vacuum ultraviolet (VUV), extreme ultraviolet (EUV) and soft X-ray spectral ranges. The schematic of the optical layout of the Kosmos Metrological Station which is the beamline No. 10 at the VEPP-4M synchrotron, is presented in Fig. 3. The synchrotron radiation from a VEPP-4M bending magnet goes through an entrance set of slits, then comes through one spectral filter from a filter set (e.g. Al foil for EUV spectral range, and $\mathrm{MgF}_{2}$ in case of measurements in the VUV), then comes into a plane diffraction grating monochromator, and after a set of exit slits goes into the experimental vacuum chamber. The Kosmos Metrological Station is equipped with a differential pumping system to ensure high vacuum in the vacuum tube for synchrotron radiation transportation (starting from the VEPP-4M bending magnet and finishing near the set of exit slits) with pressure of residual gases of the order of $10^{-7} \mathrm{~Pa}$, and to keep the residual gases pressure of the order of $10^{-4} \mathrm{~Pa}$ in the main experimental vacuum chamber.

The plane diffraction grating monochromator consists of two optical elements mounted in parallel and organized to have the possibility to be rotated simultaneously. The monochromator has a plane mirror and a plane diffraction grating with a groove density of $300 \mathrm{~mm}^{-1}$. The operating surface of the diffraction grating is covered with gold, and each groove has a $2.5^{\circ}$ blazing angle. The light incident on the grating is reflected and dispersed, and scanning the wavelength is fulfilled via simple rotation of the plane grating, while keeping the plane mirror position fixed. The plane mirror in our experiments has been arranged to have an angle of $24^{\circ}$ between the incident synchrotron radiation and the plane mirror surface. The monochromator in the scheme operates at a constant zero deviation angle. The distance between the center of the diffraction grating and the set of exit slits is $1.4 \mathrm{~m}$. We used a $\mathrm{MgF}_{2}$ spectral filter at the stage of the calibration in the VUV spectral range to cut all the radiation with wavelengths shorter than $112 \mathrm{~nm}$. When dealing with the EUV radiation, we used an aluminum foil $(0.3 \mu \mathrm{m}$ in width) or a zirconium foil ( $0.4 \mu \mathrm{m}$ in width) as a spectral filter. 


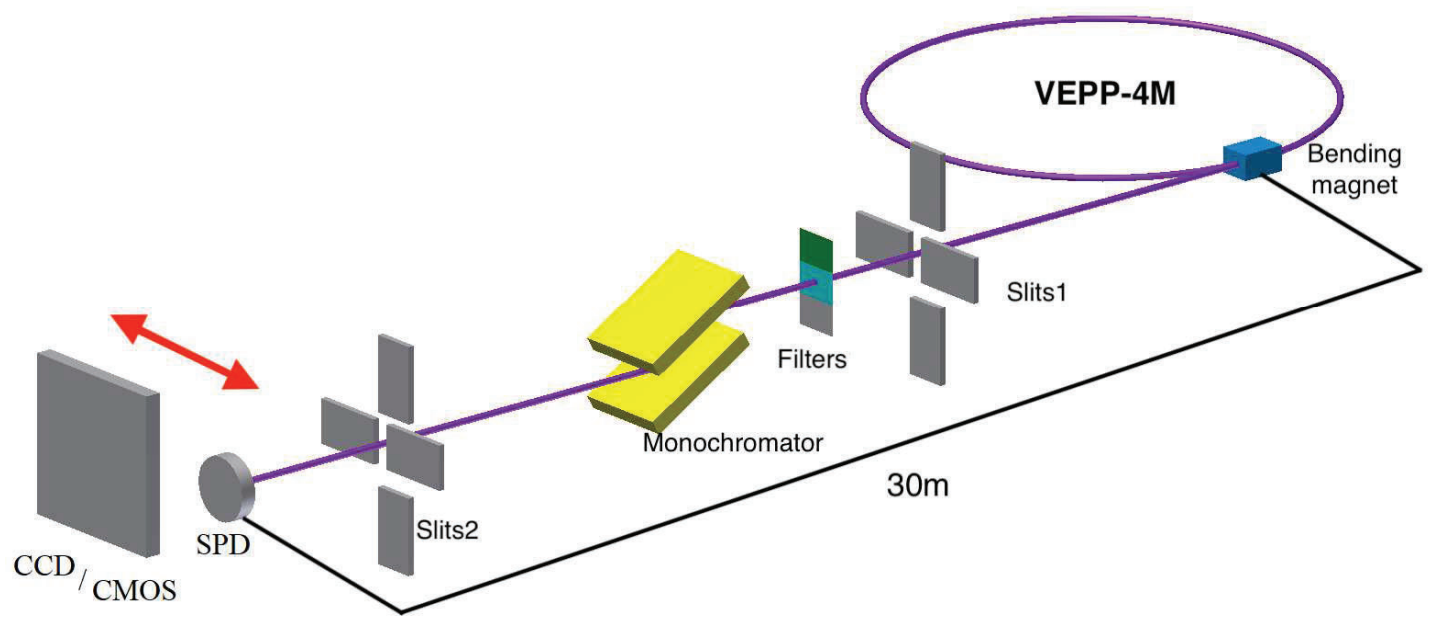

Figure 3. Schematic view of the Kosmos Metrological Station beamline. The central part is a plane grating monochromator.

The spectral sensitivity measurements of the CCD272-64 and GSENSE400BSI-GP CMOS sensors were conducted in the following manner. We selected several wavelengths in each spectral waveband of interest (115-310 nm in the VUVUV and 17-58 $\mathrm{nm}$ in the EUV) and integrated the photon flux incident on the sensor at a selected wavelength over a specified period of time. The interchangeability of the sensors in the main experimental vacuum chamber is assured by a set of mechanical translation stages, so this makes it possible to measure the integrated monochromatic photon flux incident on the CCD, on the CMOS and on the reference detector in a single cycle of measurements conducted within a relatively short period of time (within several minutes). The photon flux coming from the VEPP-4M synchrotron storage ring is proportional to the VEPP-4M electric current value that had been recorded every 30 seconds during the whole experiment.

We have used an absolutely calibrated silicon photodiode SPD as a reference detector in all series of the measurements. The absolute calibration procedure with the SPD photodiode ${ }^{18}$ was carried out twice in 2005 and 2017 at PhysicalischTechnische Bundesanstalt (PTB, Germany). The absolute calibration accuracy was $1 \%$ in both cases. The second calibration had shown that the SPD spectral sensitivity had slightly degraded in the 110-200 nm spectral interval (a minor relative fall of $\sim 5 \%$ in 12 years was reported), while in other spectral ranges the SPD sensitivity has remained stable. In our experiments, SPD was used to measure the distribution of the photon numbers into different spectrally narrow monochromatic portions of the radiation coming into the main experimental vacuum chamber after the set of exit slits (Fig. 3). This photon number distribution was measured by SPD, several times for each case of the entrance filters employed, both before and after every experimental measurement series. The selection of the spectrally narrow monochromatic portion of the synchrotron radiation was fulfilled by rotation of the reflective diffraction grating in the monochromator to a specific angle corresponding to the wavelength desired. The monochromatization level in the scheme is about $1 \mathrm{~nm}$ in VUV and about $0.1 \mathrm{~nm}$ in EUV. These values ensure better than $1 \%$ wavelength accuracy.

As both CCD and CMOS sensors give the measured values in each pixel in relative counts, it is valuable to know the Conversion Factor (CF) for each sensor in order to know the corresponding number of the electron-hole pairs generated per each count indicated by the sensor. In our case, the $\mathrm{CF}$ is equal to $0.775 \mathrm{e}^{-} /$count for CCD272-64. As for the GSENSE400BSI-GP CMOS sensors, there were two gain options available, namely Low Gain (LG) and High Gain (HG) modes with slightly different CF for the LB-SA, LB-WA, SB-SA and SB-WA areas of the sensors. The average

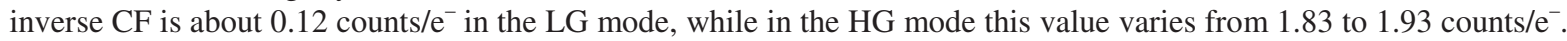

Knowing the $\mathrm{CF}$ and thus the integrated number of the charge carriers generated by the incident radiation of a known specific wavelength in the sensor, we can calculate the spectral sensitivity of the sensor by a simple division of this number of charge carriers over the integrated photon number incident on the sensor during the integration time. The results are given as a plot in terms of $\mathrm{e}^{-}$/photon versus the radiation wavelength. In order to calculate the quantum efficiency of a sensor, we divided its spectral sensitivity over the model theoretical spectral sensitivity which is calculated as a ratio of a photon energy of the monochromatic radiation and a value of $3.5 \mathrm{eV}$ which corresponds to the 
energy band gap in silicon, i. e. the energy required to generate an electron-hole pair in silicon at normal conditions. We have employed this approach before to evaluate the quantum efficiency of other laboratory CCDs ${ }^{19,20}$ which have the quantum efficiency values of $\sim 50 \%$ in the EUV spectral range and less than $20 \%$ in the VUV.

\section{EXPERIMENTAL RESULTS AND COMPARISON}

We have measured the spectral sensitivity and the quantum efficiency of all the four differently pretreated areas of the GSENSE400BSI-GP CMOS sensors in the 115-300 nm (VUV) and in the 10-58 nm (EUV) spectral ranges. The results of the measurements in VUV are presented in Fig. 4. The data in the plots show a clearly better performance of the SB (small boron implantation dose) CMOS sensor against the LB (large boron implantation dose) CMOS sensor in the whole 115-300 nm VUV spectral range, with slight, but noticeable improvement of the spectral sensitivity for the SA (strongly annealed) areas of the sensors against the WA (weakly annealed) areas. The quantum efficiency of the SB-SA CMOS area reaches $20 \%$ at a $120 \mathrm{~nm}$ wavelength, with a gradual decrease to $10 \%$ in the vicinity of $\lambda=250 \mathrm{~nm}$. The SBWA area has the highest quantum efficiency value of $16.5 \%$ at the wavelength of $130 \mathrm{~nm}$. Both areas of the SB CMOS sensor show quantum efficiency values of $10 \%$ and higher in the whole VUV spectral range. On the contrary, both areas of the LB CMOS sensor demonstrate low quantum efficiency values of $4 \%$ and lower, which are within two error bars from zero level, so, the LB CMOS sensor is not recommended to be used for the VUV spectral range.
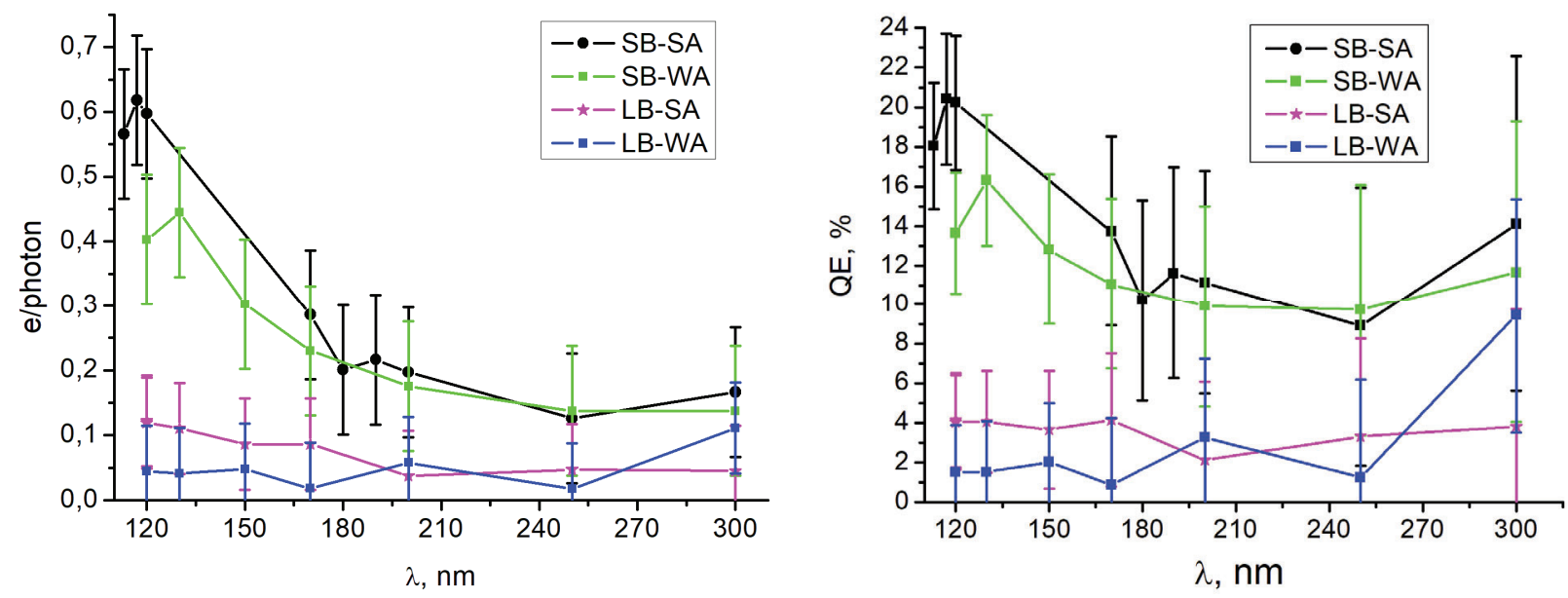

Figure 4. Experimental results of the CMOS spectral sensitivity (left) and the CMOS quantum efficiency (right) in VUV.

Along with the VUV dedicated GSENSE400BSI-GP CMOS sensors, we also measured the spectral sensitivity and the quantum efficiency of a commercially available TVISB CMOS sensor which is optimized for the best performance in the UV-visible spectral range. The result of the spectral sensitivity comparison between the GSENSE400BSI-GP CMOS sensors and the TVISB CMOS sensor in VUV is presented in the left part of Fig. 5. We can report a better performance of all the GSENSE400BSI-GP CMOS sensors against the TVISB sensor in the $115-150 \mathrm{~nm}$ spectral range, with SB CMOS having higher sensitivity than TVISB in the $115-180 \mathrm{~nm}$ spectral range. On the other hand, we see that for NUV radiation with wavelengths $\lambda>200 \mathrm{~nm}$ the TVISB CMOS is evidently better than all the GSENSE400BSI-GP CMOS sensors.

The right part of Fig. 5 presents the quantum efficiency plots for both CCD272-64 (with quantum yield correction) of the WSO-UV space project and all the four differently pretreated areas of the GSENSE400BSI-GP CMOS sensors in the 115-310 nm range. We can state that the CCD performance is better than that of the LB-WA, LB-SA and SB-WA CMOS areas in the whole VUV spectral range, while the SB-SA CMOS area has higher quantum efficiency in the 115$150 \mathrm{~nm}$ range. It is worth mentioning that in Fig. 5 we have presented quantum efficiency only for the uncoated area of the CCD, while the other area of the CCD with a specially designed anti-reflection coating has even higher quantum efficiency in the $170-310 \mathrm{~nm}$ spectral range ${ }^{16}$. It means that among measured sensors, CCD now is more preferable than the CMOS sensors in VUV. 

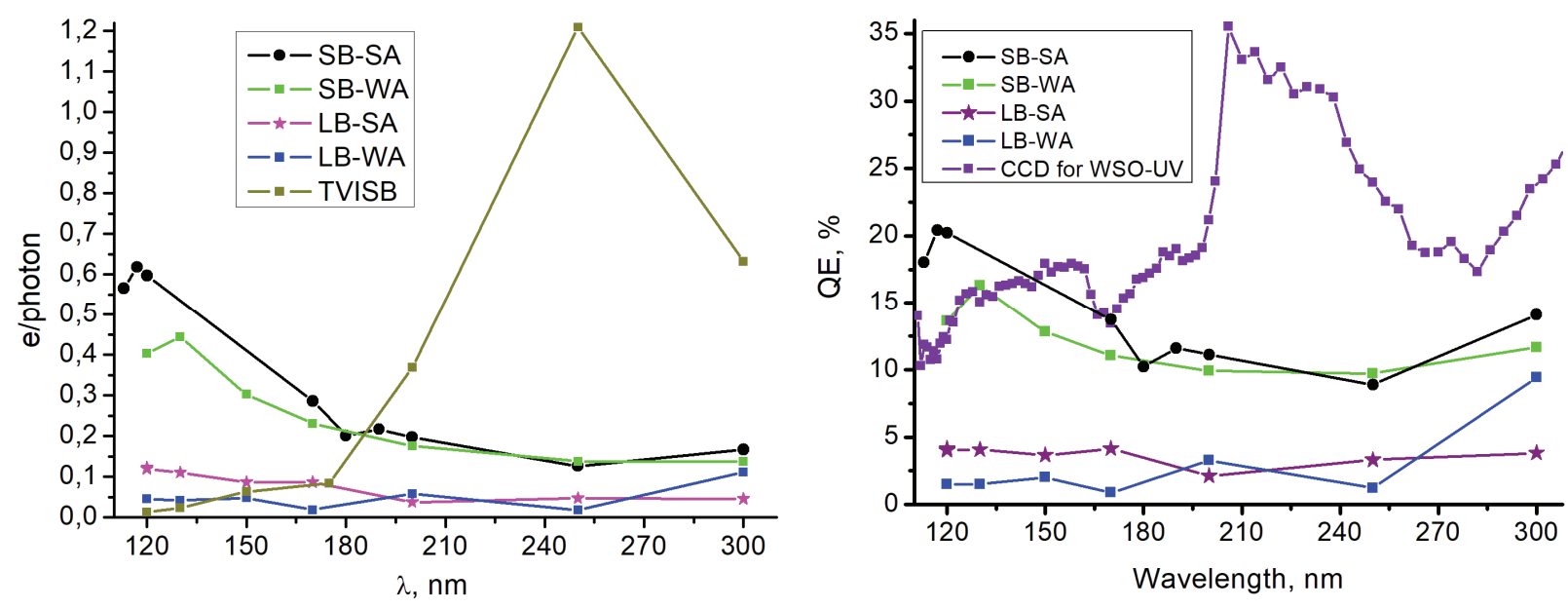

Figure 5. Comparison of the GSENSE400BSI-GP CMOS and TVISB CMOS spectral sensitivity in the VUV (left); comparison of the CCD272-64 for the WSO-UV and GSENSE400BSI-GP CMOS quantum efficiency in VUV (right).

In the extreme ultraviolet (EUV) spectral range, we have measured the spectral sensitivity and the quantum efficiency of the GSENSE400BSI-GP CMOS sensors and the TVISB CMOS sensor in the 10-58 nm spectral interval. We used an Al foil $(0.3 \mu \mathrm{m}$ thick $)$ as a spectral filter in the $17-58 \mathrm{~nm}$ waveband, and $\mathrm{Zr}$ foil $(0.4 \mu \mathrm{m}$ thick $)$ for the $10-17 \mathrm{~nm}$ spectral range. The data in Fig. 6 reveals that large boron implantation dose decreases the CMOS sensitivity and the quantum efficiency in EUV not so substantially as it was found for the VUV spectral range. The effect of the annealing is within the error bars for the majority of the experimental points, with slightly better performance of the SA CMOS areas against the WA areas at wavelengths $\lambda<17 \mathrm{~nm}$. Several measurement points for TVISB CMOS show a prominent advantage of all areas of the GSENSE400BSI-GP CMOS sensors against the TVISB CMOS sensor in the whole 10-58 nm EUV spectral interval. The right part of Fig. 6 could seem abnormal with the quantum efficiency values higher than $100 \%$ at some wavelengths for all areas of the GSENSE400BSI-GP CMOS sensors. Such unexpected high quantum efficiency values larger than unity have been experimentally observed in several cases, including silicon solar cells ${ }^{21}$. The explanation of these high values may lie in a theory of impact ionization by hot electrons in silicon, when the standard $3.5 \mathrm{eV}$ photon energy could create more than a single electron-hole pair due to the carrier multiplication by the hot charge carrier effects. Ultraviolet, VUV and EUV photons are energetic enough to facilitate photon-induced impact ionization processes, so this effect is probable. Nevertheless, the new CMOS sensors have demonstrated a significantly better sensitivity to the EUV radiation than TVISB CMOS and thus they could be recommended to be used in EUV.
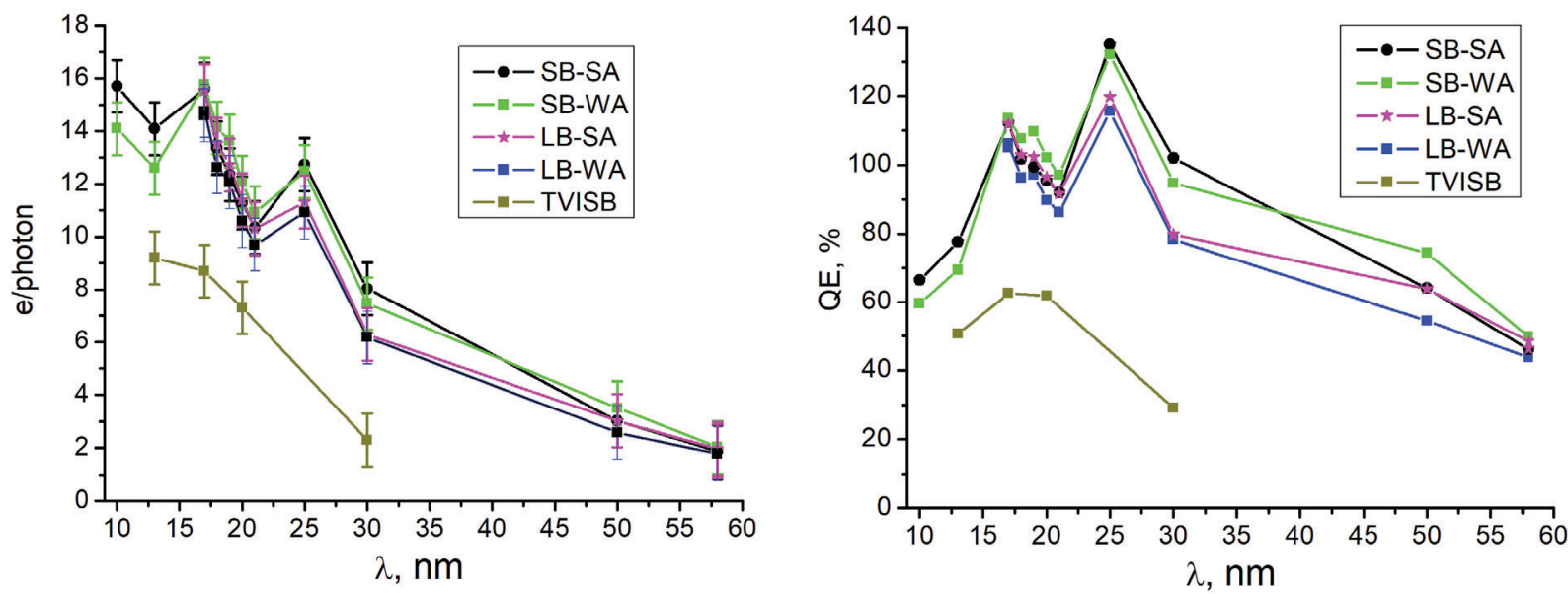

Figure 6. Experimental results of the CMOS spectral sensitivity (left) and the CMOS quantum efficiency (right) in EUV. 


\section{SUMMARY}

We have measured the spectral sensitivity and the quantum efficiency of the VUV dedicated GSENSE400BSI-GP CMOS sensors and the custom UV-optimized sealed enclosure containing a custom UV-optimized CCD272-64 sensor which will be used in the WSO-UV space project. We have utilized the synchrotron radiation from the VEPP-4M storage ring to carry out the measurements in two broad spectral ranges: VUV (115-310 nm) and EUV (10-58 nm).

The results of the measurements have shown that a large boron implantation dose decreases the CMOS sensitivity in both VUV and EUV spectral range, while strong annealing could slightly increase the spectral sensitivity of CMOS. The new GSENSE400BSI-GP CMOS sensors have higher quantum efficiency in the $10-58 \mathrm{~nm}$ spectral interval, compared to the commercially available TVISB CMOS sensor which is optimized for the best performance in UV-visible wavelengths. As for the VUV spectral range, the CMOS sensors could have an improved spectral sensitivity in the 115$180 \mathrm{~nm}$ range, compared to the TVISB CMOS sensor, while both CCD and TVISB CMOS sensors are more sensitive to radiation with wavelengths $\lambda>180 \mathrm{~nm}$. We may conclude that among measured devices, the CCD sensor is more preferable in the 180-310 nm spectral range rather than the new CMOS sensors, but both technologies are far away from the $100 \%$ quantum efficiency limit, so both of them have the potential to improve the EUV and VUV sensitivity, for example, with the use of special coatings and treatments of the back surface of the sensors.

\section{ACKNOWLEDGEMENTS}

We express our gratitude to the Teledyne e2v and RAL Space companies for the production of the custom CCD272-64, custom enclosures and low readout noise electronics for the WSO-UV space project.

We are thankful to GPixel company for the great possibility to measure the quantum efficiency of the GSENSE400BSIGP CMOS sensors with different pretreatment.

We highly acknowledge the VEPP-4M operating team for their sustainable technical support and for the excellent synchrotron performance.

\section{REFERENCES}

[1] Weckler, G. P., "A silicon photodevice to operate in a photon flux integration mode," Proc. IEDM, 38-39 (1965).

[2] Weckler, G. P., "Operation of p-n junction photodetectors in a photon-flux integrating mode," IEEE J. Solid State Circuits 2 (3), 65-73 (1967).

[3] Noble, P. J. W., "Self-scanned silicon image detector arrays," IEEE Trans. Electron. Devices 15 (4), 202-209 (1968).

[4] Boyle, W. S. and Smith, G. E., "Charge coupled semiconductor devices," Bell Syst. Tech. J. 49 (4), $587-593$ (1970).

[5] Amelio, G. F., Tompsett, M. F. and Smith, G. E., "Experimental verification of the Charge Coupled Device concept," Bell Syst. Tech. J. 49 (4), 593-600 (1970).

[6] Smith, G. E., "Nobel Lecture: The invention and early history of the CCD," Rev. Mod. Phys. 82 (3), $2307-2312$ (2010).

[7] Shortes, S. R., Chan, W. W., Rhines, W. C., Barton, J. B. and Collins, D. R., "Development of a thinned backside-illuminated charge-coupled device imager," Proc. IEDM, 415 (1973).

[8] Shortes, S. R., Chan, W. W., Rhines, W. C., Barton, J. B. and Collins, D. R., "Characteristics of thinned backside-illuminated charge-coupled device imagers," Appl. Phys. Lett. 24 (11), 565-567 (1974).

[9] Fossum, E. R., “Active Pixel Sensors: Are CCDs dinosaurs?” Proc. SPIE 1900, 2-14 (1993).

[10] Fossum, E. R., "Ultra low power imaging systems using CMOS technology," Proc. SPIE 2267, 107-111 (1994).

[11]Fossum, E. R. and Hondongwa, D. B., "A Review of the Pinned Photodiode for CCD and CMOS Image Sensors," IEEE J. Electron Dev. Soc. 2 (3), 33-43 (2014).

[12] Shugarov, A., Savanov, I., Sachkov, M., Jerram, P., Moody, I., Pool, P., Turner, P., Pittock, R., Kuzin, S. and Waltham, N., "UV detectors for spectrographs of WSO-UV project," Astrophys. Space Sci. 354, 169-175 (2014). 
[13] Hayes-Thakore, C., Spark, S., Pool, P., Walker, A., Clapp, M., Waltham, N. and Shugarov, A., "Sensor system development for the WSO-UV (World Space Observatory-Ultraviolet) space-based astronomical telescope," Proc. SPIE 9639, 96390U (2015).

[14] Sachkov, M., Shustov, B. and Gómez de Castro, A. I., "WSO-UV project," Adv. Space Res. 53 (6), $990-995$ (2014).

[15] Shustov, B., Gómez de Castro, A. I., Sachkov, M., Moisheev, A., Kanev, A., López-Santiago, J., Malkov, O., Nasonov, D., Belén Perea, G., Sánchez, N., Savanov, I., Shugarov, A., Sichevskiy, S., Vlasenko, O. and Yaňez, J., "WSO-UV progress and expectations," Astrophys. Space Sci. 354, 155-161 (2014).

[16] Vishnyakov, E. A., Shugarov, A. S., Ivlyushkin, D. V., Zavertkin, P. S., Nikolenko, A. D., Chervinskiy, V. I., Pertsov, A. A., Erkhova, N. F., and Kuzin, S. V., "Evaluation of CCD detector absolute responsivity with the aid of synchrotron radiation," AIP Conf. Proc. 2299, 060007 (2020).

[17] Nikolenko, A. D., Avakyan, S. V., Afanas'ev, I. M., Voronin, N. A., Kovalenko, N. V., Legkodymov, A. A., Lyakh, V. V. and Pindyurin, V. F., "Kosmos Station: Application of Synchrotron Radiation from the VEPP-4M Storage Ring for Metrological Measurements in the VUV and Soft X-ray Ranges,” J. Surf. Inv. X-Ray, Synchr. Neutr. Techn. 6 (3), 388-393 (2012).

[18] Scholze, F., Klein, R. and Müller, R., "Characterization of detectors for extreme UV radiation," Metrologia 43 (2), S6-S10 (2006).

[19] Vishnyakov, E. A., Kirichenko, A. S., Reva, A. A., Rizvanov, A. A., Plastinin, Ju. A. and Kuzin, S. V., "Spectral calibration of CCDs and multilayer filters intended for future space applications," Proc. SPIE 9905, Space Telescopes and Instrumentation 2016: Ultraviolet to gamma ray, 99053G (2016).

[20] Vishnyakov, E. A., Shcherbakov, A. V., Pertsov, A. A., Polkovnikov, V. N., Pestov, A. E., Pariev, D. E. and Chkhalo, N. I., "High-aperture monochromator-reflectometer and its usefulness for CCD calibration," Proc. SPIE 10235, EUV and X-ray Optics: Synergy between Laboratory and Space V, 102350W (2017).

[21] Kolodinski, S., Werner, J. H., Wittchen, T. and Queisser, H. J., "Quantum efficiencies exceeding unity due to impact ionization in silicon solar cells,” Appl. Phys. Lett. 63 (17), 2405-2407 (1993). 